\title{
ON ILL-POSEDNESS FOR THE ONE-DIMENSIONAL PERIODIC CUBIC SCHRODINGER EQUATION
}

\author{
LuC Molinet
}

\begin{abstract}
We prove the ill-posedness in $H^{s}(\mathbb{T}), s<0$, of the periodic cubic Schrödinger equation in the sense that the flow-map is not continuous from $H^{s}(\mathbb{T})$ into itself for any fixed $t \neq 0$. This result is slightly stronger than the one in [7] where the discontinuity of the solution map is established. Moreover our proof is different and clarifies the ill-posedness phenomena. Our approach relies on a new result on the behavior of the associated flow-map with respect to the weak topology of $L^{2}(\mathbb{T})$.
\end{abstract}

\section{Introduction}

In this work we are interested in the failure of well-posedness in some spaces of rough functions for the one dimensional cubic Schrödinger equation in the periodic setting. Throughout this paper we will say that a Cauchy problem is (locally) wellposed in some normed function space $X$ if, for any initial data $u_{0} \in X$, there exist a radius $R>0$, a time $T>0$ and a unique solution $u$, belonging to some space-time function space continuously embedded in $C([0, T] ; X)$, such that for any $t \in[0, T]$ the map $u_{0} \mapsto u(t)$ is continuous from the ball of $X$ centered at $u_{0}$ with radius $R$ into $X$. A Cauchy problem will be said to be ill-posed if it is not well-posed.

Let us recall that the cubic Schrödinger equation reads

$$
i u_{t}+u_{x x}+\gamma|u|^{2} u=0,
$$

where $\gamma \neq 0$ is a real number. In the one-dimensional periodic setting, $u=u(t, x)$ is a function from $\mathbb{R} \times \mathbb{T}$ to $\mathbb{C}$ where $\mathbb{T}:=\mathbb{R} / 2 \pi \mathbb{Z}$. Note that this equation is completely integrable (cf. [1]) even if we will not directly exploit this particularity.

It is well-known that this equation enjoys two symmetries. Namely, the dilation symmetry : $u(t, x) \mapsto \lambda u\left(\lambda^{2} t, \lambda x\right)$ and the Galilean invariance : $u(t, x) \mapsto$ $e^{i \alpha x / 2} e^{-i \alpha^{2} t / 4} u(t, x-\alpha t)$. Recall that to each equation that enjoys a dilation symmetry, one can associate a so called critical scaling Sobolev index $s_{c}$. This is the index of the homogeneous Sobolev space on the line whose semi-norm remains invariant by the dilation symmetry of the equation. It is commonly conjectured (at least when working on $\mathbb{R}$ or $\mathbb{T}$ ) that an equation must be ill-posed in $H^{s}$ as soon as $s<s_{c}$ (However, as far as the author knows, there exists no general proof of this fact). On the other hand, one can expect that the equations are well-posed above $s_{c}$. This is indeed the case for a large class of parabolic equations as the dissipative Burgers equation, on $\mathbb{R}$ or $\mathbb{T}$, and for dispersive equations as the nonlinear Schrödinger equation on $\mathbb{R}^{N}$ and the generalized Korteweg-de Vries equation on $\mathbb{R}$ with $L^{2}$-critical or $L^{2}$-supercritical nonlinearity.

Received by the editors January 7, 2008. 
For the cubic Schrödinger equation $s_{c}=-1 / 2$ and well-posedness is known to hold in $H^{s}(\mathbb{R})$ and $H^{s}(\mathbb{T})$ for $s \geq 0$ (see [10] on $\mathbb{R}$ and [3] on $\mathbb{T}$ ). However, as we mentioned, there exists another symmetry, the Galilean invariance, for which the critical index is 0 . This suggests that this equation may be ill-posed in $H^{s}$ as soon as $s<0$. A first step in this direction was due to Bourgain $([4])$ who observed that for a wide class of dispersive equations there exists a critical index $s_{c}^{\infty}>s_{c}$ below which the flow-map of the equation (if it exists) cannot be smooth whereas it is real analytic ${ }^{1}$ above. For the cubic Schrödinger equation on $\mathbb{R}$ or $\mathbb{T}$, this approach shows that the flow-map (if it exists) cannot be of class $C^{3}$ as soon as $s<0$, i.e. $s_{c}^{\infty}=0$. A second step was initiated by Kenig-Ponce and Vega ([9]) who discovered that more qualitative ill-posedness phenomena can occur above $s_{c}$ for dispersive equations on the line. They observed that the lack of uniform continuity on bounded set of the flow-map associated with some canonical focusing dispersive equations, including the cubic focusing Schrödinger equation, can be proven below $s_{c}^{\infty}$ by using a two parameter family of exact solutions. Using the same idea, Burq-Gerard-Tzvetkov (cf. [5]) noticed that the one-dimensional periodic cubic Schrödinger equation (1) cannot be uniformly continuous on bounded set below $L^{2}$. The proof is based on the fact that the solution emanating from the initial data $\alpha e^{i n x}$ is explicitly given by

$$
u(t, x)=\alpha \exp \left(-i t\left(n^{2}-\gamma \alpha^{2}\right)\right) e^{i n x} .
$$

In the same paper, they proved the same type of results on the sphere by constructing approximate solutions.

In [6], Christ-Colliander and Tao also exploited the construction of approximate solutions in order to extend the result of [9] in the case of defocusing equations as well as in the periodic case. Finally, very recently, they proved in [7] an ill-posedness result for the one dimensional periodic cubic Schrödinger equation below $L^{2}(\mathbb{T})$ in the sense that the solution map associated with this equation (defined on $L^{2}(\mathbb{T})$ ) cannot be continuously extended in $H^{s}(\mathbb{T})$ with values in $C\left([0, T] ; H^{s}(\mathbb{T})\right)$ as soon as $s<0$. The main point is the construction of approximate solutions corresponding to initial data that are supported on two different modes : the mode 0 plus a mode going to infinity.

In this work we give a proof of the ill-posedness of $(1)$ below $L^{2}(\mathbb{T})$ in the strong sense stated in the beginning of the introduction. Our improvement with respect to the result of [7] is that we show the discontinuity of the flow-map for any fixed $t \neq 0$ instead of showing the discontinuity of the the solution map. Moreover our approach is different and relies on the lack of continuity for the $L^{2}(\mathbb{T})$-weak topology of the flow-map associated with this equation. It does not exploit the existence of exact or approximate solutions. The main idea is to notice that the nonlinear term of the cubic Schrödinger equation can be split into two terms. One which enjoys a good behavior with respect to the weak convergence in the resolution space. The other which is of the form $g\left(\int_{\mathbb{T}}|u|^{2}\right) u$. Since $\int_{\mathbb{T}}|u|^{2}$ is a constant of the motion for (1), this will lead to

\footnotetext{
${ }^{1}$ For dispersive periodic equations whose nonlinear term is of the form $u^{q} u_{x}$ the smoothness of the flow-map holds not for the original equation but for the equation satisfied by $\tilde{u}(t, x)=u\left(t, x-\int_{0}^{t} f u^{q}\right)$. Therefore the non-smoothness of the flow-map has to be shown on this last equation. Note, however, that for $q=1$, since the mean-value of $u$ is conserved, the smoothness of the flow-map associated with $\tilde{u}$ ensures the smoothness of the flow-map associated with the original equation on hyperplans of functions with a given mean-value.
} 
the lack of weak continuity in $L^{2}(\mathbb{T})$ for the flow-map. Note that this approach does not work for the equation posed on the line. Indeed, it was recently proved in [8] that the flow-map associated with the cubic Schrödinger equation is continuous in $L^{2}(\mathbb{R})$ endowed with the weak topology, even if $s_{c}^{\infty}=0$. It is also interesting to notice that our result uses in a crucial way the well-posednes of the equation in $L^{2}(\mathbb{T})$ which is the critical space for $C^{\infty}$-well-posedness. Let us note that the use of the well-posedness result in the critical space in order to prove ill-posedness appears also in [2]. Finally we would like to mention that we strongly believe that the approach developed in this paper can also lead to the ill-posedness of the periodic Benjamin-Ono equation below $L^{2}(\mathbb{T})$.

1.1. Main results. Our ill-posedness result is a straightforward corollary of the following theorem.

Theorem 1.1. Let $\left\{u_{0, n}\right\}$ be a sequence of $L^{2}(\mathbb{T})$ converging weakly, but not strongly, to $u_{0}$ in $L^{2}(\mathbb{T})$ and let $\left\{u_{n}\right\}$ be the sequence of associated solutions of the cubic Schrödinger equation (1). For any adherence value $\alpha^{2} \in \mathbb{R}_{+}$of $\int_{\mathbb{T}}\left|u_{0, n}\right|^{2}$ (at least one such value must be different from $\int_{\mathbb{T}}\left|u_{0, n}\right|^{2}$ ) and any increasing sequence of integers $\left\{n_{k}\right\}$ such that $\int_{\mathbb{T}}\left|u_{0, n_{k}}\right|^{2}$ converges to $\alpha^{2}$, the sequence $u_{n_{k}}(t)$ converges weakly in $L^{2}(\mathbb{T})$ to

$$
v_{\alpha}(t)=\exp \left(\frac{i \gamma}{\pi}\left(\alpha^{2}-\int_{\mathbb{T}}\left|u_{0}\right|^{2}\right) t\right) u(t)
$$

for all $t \in \mathbb{R}$, where $u \in C\left(\mathbb{R} ; L^{2}(\mathbb{T})\right)$ is the (unique) solution to (1) emanating from $u_{0}$.

Remark 1.2. As we already mentioned, in [5] Burg-Gerard-Tzvetkov studied sequences of initial data that are supported on one single mode going to infinity whereas in [7] Christ-Colliander-Tao studied a sequence of initial data that are supported on two different modes : the mode 0 plus a mode going to infinity. These sequences are bounded in $H^{s}(\mathbb{T}), s<0$, but not in $L^{2}(\mathbb{T})$. Therefore, we cannot re-examine them with the help of Theorem 1.1. However, we can observe what happens with sequences of initial data having the same support properties.

Sequences of the type $u_{0, n}=\alpha_{n} e^{i n x}$, with $\left\{\alpha_{n}\right\}$ bounded, converge weakly to 0 in $L^{2}(\mathbb{T})$ and Theorem 1.1 ensures that the corresponding sequences of emanating solutions tend weakly in $L^{2}(\mathbb{T})$ to 0 for any $t \in \mathbb{R}$. Of course this implies the strong convergence to 0 in any $H^{s}$ for $s<0$. It is thus clear that this type of counterexamples cannot disprove the continuity of the flow-map.

Observe now what happens with a sequence of the type $u_{0, n}=\beta_{1}+\beta_{2} e^{i n x}$. This sequence tends weakly to $u_{0} \equiv \beta_{1}$ in $L^{2}(\mathbb{T})$ and for any $n \in \mathbb{N}^{*}$ it holds $\left\|u_{0, n}\right\|_{L^{2}}^{2}=$ $2 \pi\left(\left|\beta_{1}\right|^{2}+\left|\beta_{2}\right|^{2}\right)$. The solution of (1) emanating from $u_{0}=\beta_{1}$ is explicitly given by $u(t)=\beta_{1} e^{i t \gamma\left|\beta_{1}\right|^{2} t}$. On the other hand, Theorem 1.1 ensures that the sequence of emanating solutions $\left\{u_{n}\right\}$ tends weakly in $L^{2}(\mathbb{T})$ and thus strongly in $H^{s}(\mathbb{T}), s<0$, for any fixed $t \in \mathbb{R}$ toward $v(t)=\beta_{1} e^{2 i \gamma\left(\left|\beta_{1}\right|^{2}+\left|\beta_{2}\right|^{2}\right) t}$. Since

$$
|v(t)-u(t)| \geq\left|\beta_{1}\right|\left|1-e^{i 2 \gamma\left|\beta_{2}\right|^{2} t}\right| \geq|\gamma|\left|\beta_{1}\right|\left|\beta_{2}\right|^{2} t
$$

for $t>0$ small enough, this ensures that the flow-map is not continuous at $u_{0}=\beta_{1}$ for the $H^{s}(\mathbb{T})$-topology as soon as $s<0$. 
Remark 1.3. We strongly believe that Theorem 1.1 is interesting by its own and not only as a tool to prove ill-posedness. For instance, in a forthcoming paper we will use a variant of this theorem to prove the existence of a compact global attractor in $L^{2}(\mathbb{T})$ for the weakly damped Schrödinger equation

$$
u_{t}+i u_{x x}+\varepsilon u+i|u|^{2} u=f
$$

where $\varepsilon>0$ is the damping parameter and where the external forcing $f$, that is independent of $t$, belongs to $L^{2}(\mathbb{T})$. See [8] for the case on the line.

As a consequence of Theorem 1.1 we get that the flow-map of (1) is not continuous for the weak $L^{2}(\mathbb{T})$-topology. The ill-posedness, in the strong sense stated in the introduction, of (1) in $H^{s}(\mathbb{T}), s<0$, follows directly (see Remark 1.5 below).

Corollary 1.4. For any $t \neq 0$, the flow-map associated with the periodic cubic Schrödinger equation (1) is discontinuous as a map from $L^{2}(\mathbb{T})$, equipped with its weak topology, into the space of distributions $\left(C^{\infty}(\mathbb{T})\right)^{*}$ at any point $u_{0} \in L^{2}(\mathbb{T})$ different from the origin.

Remark 1.5. Let $B$ be any topological space continuously embedded in the space of distributions $\left(C^{\infty}(\mathbb{T})\right)^{*}$ and such that $L^{2}(\mathbb{T})$ is compactly embedded in B. Note that this is obviously the case of $H^{s}(\mathbb{T})$ as soon as $s<0$. Corollary 1.4 proves the discontinuity of the map $u_{0} \mapsto u(t)$ from $B$ into $B$ at any $u_{0} \in L^{2}(\mathbb{T})$ different from the origin and for any fixed $t \neq 0$.

Proof of Corollary 1.4. Let $u_{0} \in L^{2}(\mathbb{T})$ different from 0 and let $\left\{\phi_{n}\right\} \subset L^{2}(\mathbb{T})$ be a sequence such that $\phi_{n} \rightarrow 0$ in $L^{2}(\mathbb{T})$ and $\left\|\phi_{n}\right\|_{L^{2}}^{2} \rightarrow 2 \pi \theta^{2}, \theta \in \mathbb{R}^{*}$, as $n$ goes to infinity (one can take for instance $\phi_{n}=\theta e^{i n x}$ ). Setting $u_{0, n}=u_{0}+\phi_{n}$, we get that $u_{0, n} \rightarrow u_{0}$ in $L^{2}(\mathbb{T})$ and $\left\|u_{0, n}\right\|_{L^{2}}^{2} \rightarrow\left\|u_{0}\right\|_{L^{2}}^{2}+2 \pi \theta^{2}$ as $n \rightarrow \infty$. On account of Theorem 1.1, the emanating solutions $u_{n}$ tend weakly in $L^{2}(\mathbb{T})$ for any fixed $t \in \mathbb{R}$ to $v(t, x)=e^{i 2 \gamma \theta^{2} t} u(t, x)$. Observing that

$$
|v(t, x)-u(t, x)|=\left|1-e^{i 2 \gamma \theta^{2} t}\right||u(t, x)|
$$

and that, $u_{0} \neq 0$ ensures that $u(t) \neq 0$ for any $t \in \mathbb{R}$, we infer that

$$
v(t) \neq u(t) \text { in }\left(C^{\infty}(\mathbb{T})\right)^{*}, \forall t \notin\left\{\frac{k \pi}{\gamma \theta^{2}}, k \in \mathbb{Z}\right\},
$$

Fixing $t \neq 0$ and choosing $\theta$ such that $t \notin\left\{\frac{k \pi}{\gamma \theta^{2}}, k \in \mathbb{Z}\right\}$, the discontinuity at $u_{0}$ of the map $u_{0} \mapsto u(t)$, from $L^{2}(\mathbb{T})$ equipped with its weak topology into $\left(C^{\infty}(\mathbb{T})\right)^{*}$, follows.

\section{Proof of Theorem 1.1}

Let us first introduce some notations and function spaces we will work with. For a $2 \pi$-periodic function $\varphi$, we define its space Fourier transform by

$$
\hat{\varphi}(k):=\frac{1}{2 \pi} \int_{\mathbb{T}} e^{-i k x} \varphi(x) d x, \quad \forall k \in \mathbb{Z}
$$

and denote by $V(\cdot)$ the free group associated with the linearized Schrödinger equation,

$$
\widehat{V(t) \varphi}(k):=e^{-i k^{2} t} \hat{\varphi}(k), \quad k \in \mathbb{Z} .
$$


The Sobolev spaces $H^{s}(\mathbb{T})$ for $2 \pi$-periodic functions are defined as usually and endowed with

$$
\|\varphi\|_{H^{s}(\mathbb{T})}:=\left\|\langle k\rangle^{s} \widehat{\varphi}(k)\right\|_{l^{2}(\mathbb{Z})}=\left\|J_{x}^{s} \varphi\right\|_{L^{2}(\mathbb{T})},
$$

where $\langle\cdot\rangle:=\left(1+|\cdot|^{2}\right)^{1 / 2}$ and $\widehat{J_{x}^{s} \varphi}(k):=\langle k\rangle^{s} \widehat{\varphi}(k)$.

For a function $u(t, x)$ on $\mathbb{T}^{2}$, we define its space-time Fourier transform by

$$
\hat{u}(q, k):=\mathcal{F}_{t, x}(u)(q, k):=\frac{1}{(2 \pi)^{2}} \int_{\mathbb{T}^{2}} e^{-i(q t+k x)} u(t, x), \quad \forall(q, k) \in \mathbb{Z}^{2}
$$

and define the Bourgain spaces $X^{b, s}$ and $\tilde{X}^{b, s}$ of functions on $\mathbb{T}^{2}$ endowed with the norm

$$
\|u\|_{X^{b, s}}:=\left\|\left\langle q+k^{2}\right\rangle^{b}\langle k\rangle^{s} \hat{u}\right\|_{l^{2}\left(\mathbb{Z}^{2}\right)}=\left\|\langle q\rangle^{b}\langle k\rangle^{s} \mathcal{F}_{t, x}(V(-t) u)\right\|_{l^{2}\left(\mathbb{Z}^{2}\right)}
$$

and

$$
\|u\|_{\tilde{X}^{b, s}}:=\left\|\left\langle q-k^{2}\right\rangle^{b}\langle k\rangle^{s} \hat{u}\right\|_{l^{2}\left(\mathbb{Z}^{2}\right)}=\left\|\langle q\rangle^{b}\langle k\rangle^{s} \mathcal{F}_{t, x}(V(t) u)\right\|_{l^{2}\left(\mathbb{Z}^{2}\right)} .
$$

For a function $u(t, x)$ on $\mathbb{R} \times \mathbb{T}$, we define its space-time Fourier transform by

$$
\hat{u}(\tau, \xi):=\mathcal{F}_{t, x}(u)(\tau, \xi):=\frac{1}{2 \pi} \int_{\mathbb{R} \times \mathbb{T}} e^{-i(\tau t+k x)} u(t, x) d t d x, \quad \forall(\tau, k) \in \mathbb{R} \times \mathbb{Z} .
$$

and define the Bourgain spaces $X_{\mathbb{R}}^{b, s}$ of functions on $\mathbb{R} \times \mathbb{T}$ endowed with the norm

$$
\|u\|_{X_{\mathbb{R}}^{b, s}}:=\left\|\left\langle\tau+k^{2}\right\rangle^{b}\langle k\rangle^{s} \hat{u}\right\|_{L^{2}\left(\mathbb{R} ; l^{2}(\mathbb{Z})\right)}=\left\|\langle\tau\rangle^{b}\langle k\rangle^{s} \mathcal{F}_{t, x}(V(-t) u)\right\|_{L^{2}\left(\mathbb{R} ; l^{2}(\mathbb{Z})\right)} .
$$

Finally, for $T>0$ we define the restriction in time spaces $X_{T}^{b, s}$ of functions on ]$T, T[\times \mathbb{T}$ endowed with the norm

$$
\|u\|_{X_{T}^{b, s}}:=\inf _{v \in X^{b, s}}\left\{\|v\|_{X^{b, s}}, v(\cdot) \equiv u(\cdot) \text { on }\right]-T, T[\} .
$$

whenever $0<T \leq 1$ and

$$
\|u\|_{X_{T}^{b, s}}:=\inf _{v \in X_{\mathbb{R}}^{b, s}}\left\{\|v\|_{X_{\mathbb{R}}^{b, s}}, v(\cdot) \equiv u(\cdot) \text { on }\right]-T, T[\} .
$$

whenever $T>1$.

As indicated in the introduction we will use in a crucial way the well-posedness theorem of $(1)$ in $L^{2}(\mathbb{T})$. So let us recall this theorem proven by Bourgain in [3].

Theorem 2.1. Let $s \geq 0$. For any $u_{0} \in H^{s}(\mathbb{T})$ and any $T>0$, there exists a unique solution

$$
u \in L^{4}(]-T, T[\times \mathbb{T})
$$

of (1). Moreover $u \in C\left([-T, T] ; H^{s}(\mathbb{T})\right) \cap X_{T}^{1 / 2, s}$ and the map data to solution $u_{0} \mapsto u$ is real analytic from $H^{s}(\mathbb{T})$ to $C\left([-T, T] ; H^{s}(\mathbb{T})\right)$.

This theorem principally use the linear estimates in Bourgain'spaces for the free evolution and the retarded Duhamel operator

$$
\|U(t) \varphi\|_{X_{T}^{1 / 2, s}} \leq C(T)\|\varphi\|_{H^{s}}
$$

and for any $0<\varepsilon<<1$,

$$
\left\|\int_{0}^{t} U\left(t-t^{\prime}\right) f\left(t^{\prime}\right) d t^{\prime}\right\|_{X_{T}^{1 / 2, s}} \leq C(T, \varepsilon)\|f\|_{X_{T}^{-1 / 2+\varepsilon, s}},
$$


as well as the following periodic estimate to treat the nonlinear term (see [3]):

$$
\|v\|_{L^{4}\left(\mathbb{T}^{2}\right)} \lesssim\|v\|_{X^{3 / 8,0}}, \quad \forall v \in X^{3 / 8,0} .
$$

It is worth noticing that this ensures that for $0<T<1$ it holds

$$
\|U(t) \varphi\|_{L^{4}(]-T, T[\times \mathbb{T})} \lesssim T^{1 / 8}\|\varphi\|_{L^{2}(\mathbb{T})}, \quad \forall \varphi \in L^{2}(\mathbb{T}) .
$$

The fact that the time of existence in Theorem 2.1 can be chosen arbitrarly large follows from the conservation of the $L^{2}$-norm of the solution. Note finally that excepting the fact that the solution belongs to $X_{T}^{1 / 2, s}$, the remaining of the theorem can be proven for small data by using only the following Zygmund's type estimate (see again [3]),

$$
\|U(t) \varphi\|_{L^{4}\left(\mathbb{T}^{2}\right)} \lesssim\|\varphi\|_{L^{2}(\mathbb{T})},
$$

and $T T^{*}$ classical arguments.

As we indicated in the introduction, we are going to split the nonlinear term $|u|^{2} u$ into different parts. So let us denote by $g(\cdot)$ the trilinear operator

$$
g(u, v, w):=\bar{u} v w
$$

and decompose it in the following way :

$$
\begin{aligned}
g(u, v, w)= & \sum_{k_{1}, k_{2}, k_{3} \in \mathbb{Z}} \widehat{\widehat{u}}\left(k_{1}\right) \widehat{v}\left(k_{2}\right) \widehat{w}\left(k_{3}\right) e^{i\left(k_{1}+k_{2}+k_{3}\right) x} \\
= & \sum_{k_{1}, k_{3} \in \mathbb{Z}} \widehat{\bar{u}}\left(k_{1}\right) \widehat{v}\left(-k_{1}\right) \widehat{w}\left(k_{3}\right) e^{i k_{3} x} \\
& +\sum_{k_{1}, k_{2} \in \mathbb{Z}} \widehat{\bar{u}}\left(k_{1}\right) \widehat{v}\left(k_{2}\right) \widehat{w}\left(-k_{1}\right) e^{i k_{2} x} \\
& -\sum_{k \in \mathbb{Z}} \widehat{\widehat{u}}(k) \widehat{v}(-k) \widehat{w}(-k) e^{-i k x} . \\
& +\sum_{\substack{k_{1}, k_{2}, k_{3} \in \mathbb{Z} \\
\left(k_{1}+k_{2}\right)\left(k_{1}+k_{3}\right) \neq 0}} \widehat{\bar{u}}\left(k_{1}\right) \widehat{v}\left(k_{2}\right) \widehat{w}\left(k_{3}\right) e^{i\left(k_{1}+k_{2}+k_{3}\right) x} .
\end{aligned}
$$

Note that the three first terms of the above right-hand side correspond to the resonant part of $|u|^{2} u$ under the Schrödinger flow (see (16) below). Using that $\widehat{\bar{u}}(k)=\overline{\widehat{u}}(-k)$, it is easy to check for that, taking $u=v=w$, the sum of the two first terms gives

$$
2 \sum_{k_{1}, k_{2} \in \mathbb{Z}}\left|\widehat{u}\left(k_{1}\right)\right|^{2} \widehat{u}\left(k_{2}\right) e^{i k_{2} x}=\frac{1}{\pi}\|u\|_{L^{2}}^{2} u .
$$

On the other hand, the third term is a good term with respect to the weak convergence since its mode $k$ contains only modes $k$ or $-k$ of $u, v$ and $w$. We thus rewrite $g(u):=g(u, u, u)$ as

$$
g(u)=\frac{1}{\pi}\|u\|_{L^{2}}^{2} u+\Lambda_{1}(u, u, u)+\Lambda_{2}(u, u, u),
$$

where

$$
\Lambda_{1}(u, v, w):=\sum_{\substack{k_{1}, k_{2}, k_{3} \in \mathbb{Z} \\\left(k_{1}+k_{2}\right)\left(k_{1}+k_{3}\right) \neq 0}} \widehat{\bar{u}}\left(k_{1}\right) \widehat{v}\left(k_{2}\right) \widehat{w}\left(k_{3}\right) e^{i\left(k_{1}+k_{2}+k_{3}\right) x}
$$


and

$$
\Lambda_{2}(u, v, w):=-\sum_{k \in \mathbb{Z}} \widehat{\bar{u}}(k) \widehat{v}(-k) \widehat{w}(-k) e^{-i k x} .
$$

Observe that the conjugaison is an isometry from $L^{4}\left(\mathbb{T}^{2}\right)$ into itself and that, since $\widehat{\overline{v_{1}}}(q, k)=\overline{\widehat{v}_{1}(-q,-k)}$ for all $(q, k) \in \mathbb{Z}^{2}$, it is also an isometry from $X^{3 / 8,0}$ into $\tilde{X}^{3 / 8,0}$. It follows that (8) still holds with $X^{3 / 8,0}$ replaced by $\tilde{X}^{3 / 8,0}$ and that

$$
\left\|\mathcal{F}^{-1}\left(\left|\widehat{\overline{v_{1}}}\right|\right)\right\|_{L^{4}\left(\mathbb{T}^{2}\right)} \lesssim\left\|\overline{v_{1}}\right\|_{\tilde{X}^{3 / 8,0}}=\left\|v_{1}\right\|_{X^{3 / 8,0}}
$$

Therefore, using suitable extensions of $u, v$ and $w,(8)$ and (14), it is easy to check that for $i=1,2$ and $0<T \leq 1$,

$$
\left\|\Lambda_{i}(u, v, w)\right\|_{X_{T}^{-7 / 16,0}} \lesssim\|u\|_{X_{T}^{1 / 2,0}}\|v\|_{X_{T}^{1 / 2,0}}\|w\|_{X_{T}^{1 / 2,0}}
$$

and thus $\Lambda_{1}$ and $\Lambda_{2}$ are continuous operators from $\left(X_{T}^{1 / 2,0}\right)^{3}$ to $X_{T}^{-7 / 16,0}$. Let us now show that they are also continuous on the same spaces but equipped with their respective weak topology.

Lemma 2.2. The operator $\Lambda_{2}$ is continuous from $\left(X_{1}^{1 / 2,0}\right)^{3}$ to $X_{1}^{-7 / 16,0}$ equipped with their respective weak topology.

Proof. Since $X_{1}^{3 / 8,-1 / 3}$ is compactly embedded in $X_{1}^{1 / 2,0}$, it suffices to prove that $\Lambda_{2}$ is bounded from $\left(X_{1}^{3 / 8,-1 / 3}\right)^{3}$ into $X_{1}^{-7 / 16,-1}$. But this is straightforward. Indeed, taking extensions $v_{i} \in X^{3 / 8,-1 / 3}$ of $u_{i} \in X_{1}^{3 / 8,-1 / 3}$ such that $\left\|v_{i}\right\|_{X^{3 / 8,-1 / 3}} \leq$ $2\left\|u_{i}\right\|_{X_{1}^{3 / 8,-1 / 3}, \text { it holds }}$

$$
\begin{aligned}
\left\|\Lambda_{2}\left(u_{1}, u_{2}, u_{3}\right)\right\|_{X_{1}^{-7 / 16,-1}} & \lesssim\left\|\Lambda_{2}\left(v_{1}, v_{2}, v_{3}\right)\right\|_{X-7 / 16,-1} \\
& \lesssim \sup _{\|w\|_{X^{7 / 16,1}=1}\left|\left(w, \Lambda_{2}\left(v_{1}, v_{2}, v_{3}\right)\right)_{L^{2}\left(\mathbb{T}^{2}\right)}\right|}
\end{aligned}
$$

Setting $q=q_{1}+q_{2}+q_{3}$, (8) and (14) then ensure that

$$
\begin{aligned}
\left|\left(w, \Lambda_{2}\left(v_{1}, v_{2}, v_{3}\right)\right)_{L^{2}\left(\mathbb{T}^{2}\right)}\right| & \lesssim \sum_{\left(q_{1}, q_{2}, q_{3}, k\right) \in \mathbb{Z}^{4}}\left|\widehat{w}(q,-k)\left\|\widehat{\bar{v}_{1}}\left(q_{1}, k\right)\right\| \widehat{v_{2}}\left(q_{2},-k\right) \| \widehat{v_{3}}\left(q_{3},-k\right)\right| \\
& \lesssim\left\|\mathcal{F}^{-1}\left(\langle k\rangle^{-1 / 3}\left|\widehat{v_{1}}\right|\right)\right\|_{L^{4}\left(\mathbb{T}^{2}\right)}\left\|\mathcal{F}^{-1}\left(\langle k\rangle^{-1 / 3}\left|\widehat{v_{2}}\right|\right)\right\|_{L^{4}\left(\mathbb{T}^{2}\right)} \\
& \lesssim\left\|\mathcal{F}^{-1}\left(\langle k\rangle^{-1 / 3}\left|\widehat{v_{3}}\right|\right)\right\|_{L^{4}\left(\mathbb{T}^{2}\right)}\left\|\mathcal{F}^{-1}(\langle k\rangle|\widehat{w}|)\right\|_{L^{4}\left(\mathbb{T}^{2}\right)} \\
& \lesssim\|w\|_{X^{3 / 8,1}} \prod_{i=1}^{3}\left\|v_{i}\right\|_{X^{3 / 8,-1 / 3}} .
\end{aligned}
$$

Lemma 2.3. The operator $\Lambda_{1}$ is continuous from $\left(X_{1}^{1 / 2,0}\right)^{3}$ to $X_{1}^{-7 / 16,0}$ equipped with their respective weak topology.

Proof. For the same reasons as above it suffices to prove that $\Lambda_{1}$ is bounded from $\left(X_{1}^{7 / 16,-1 / 48}\right)^{3}$ into $X_{1}^{-7 / 16,-1}$. We proceed as in the proof of the preceding lemma by introducing extensions $v_{i}$ of $u_{i}$ such that $\left\|v_{i}\right\|_{X^{3 / 8,-1 / 3}} \leq 2\left\|u_{i}\right\|_{X_{1}^{3 / 8,-1 / 3}}$. Setting 
$k=k_{1}+k_{2}+k_{3}$, we divide the region $A:=\left\{\left(k_{1}, k_{2}, k_{3}\right) \in \mathbb{Z}^{3},\left(k_{1}+k_{2}\right)\left(k_{1}+k_{3}\right) \neq 0\right\}$ of $\mathbb{Z}^{3}$ into two regions to estimate

$$
I:=\sum_{\substack{\left(q_{1}, q_{2}, q_{3}\right) \in \mathbb{Z}^{3} \\\left(k_{1}, k_{2}, k_{3}\right) \in A}}|\widehat{w}(q, k)|\left|\widehat{\widehat{v_{1}}}\left(q_{1}, k_{1}\right)\right|\left|\widehat{v_{2}}\left(q_{2}, k_{2}\right)\right|\left|\widehat{v_{3}}\left(q_{3}, k_{3}\right)\right|
$$

- The region $A_{1}:=\left\{\left(k_{1}, k_{2}, k_{3}\right) \in A,|k| \geq \frac{1}{4} \max _{i=1,2,3}\left(\left|k_{i}\right|\right)\right\}$. In this region the result for $\Lambda_{1}$ follows exactly the same lines as for $\Lambda_{2}$.

- The region $A_{2}:=\left\{\left(k_{1}, k_{2}, k_{3}\right) \in A,|k|<\frac{1}{4} \max _{i=1,2,3}\left(\left|k_{i}\right|\right)\right\}$. In this region we first notice that $\max \left(\left|k_{1}+k_{2}\right|,\left|k_{1}+k_{3}\right|\right) \geq \frac{1}{4} \max \left(\left|k_{i}\right|\right)$ since otherwise we would have $\max \left(\left|k_{1}+k_{2}\right|,\left|k_{1}+k_{3}\right|\right)<\frac{1}{4} \max \left(\left|k_{i}\right|\right)$ which is clearly incompatible with $|k|<$ $\frac{1}{4} \max _{i=1,2,3}\left(\left|k_{i}\right|\right)$.

Setting $\sigma=q+k^{2}, \tilde{\sigma}_{1}=q_{1}-k_{1}^{2}, \sigma_{2}=q_{2}+k_{2}^{2}$ and $\sigma_{3}=q_{3}+k_{3}^{2}$ with $k=k_{1}+k_{2}+k_{3}$ and $q=q_{1}+q_{2}+q_{3}$, we get the well-known resonant relation for the cubic Schrödinger equation :

$$
\sigma-\tilde{\sigma}_{1}-\sigma_{2}-\sigma_{3}=2\left(k_{1}+k_{2}\right)\left(k_{1}+k_{3}\right) .
$$

This, combining with the fact that $\min \left(\left|k_{1}+k_{2}\right|,\left|k_{1}+k_{3}\right|\right) \geq 1$, ensure that

$$
\max \left(|\sigma|,\left|\tilde{\sigma}_{1}\right|,\left|\sigma_{2}\right|,\left|\sigma_{3}\right|\right) \gtrsim \max _{i=1,2,3}\left(\left|k_{i}\right|\right) \gtrsim \max _{i=1,2,3}\left\langle k_{i}\right\rangle .
$$

It results that

$$
\begin{aligned}
& I_{/ \mathbb{Z}^{3} \times A_{2}} \lesssim \sum_{\substack{\left(q_{1}, q_{2}, q_{3}\right) \in \mathbb{Z}^{3} \\
\left(k_{1}, k_{2}, k_{3}\right) \in A_{2}}}\langle\sigma\rangle^{1 / 16}|\widehat{w}(q, k)|\left\langle\tilde{\sigma}_{1}\right\rangle^{1 / 16}\left\langle k_{1}\right\rangle^{-1 / 48}\left|\widehat{\widehat{v_{1}}}\left(q_{1}, k_{1}\right)\right| \\
& \prod_{i=2}^{3}\left\langle\sigma_{i}\right\rangle^{1 / 16}\left\langle k_{i}\right\rangle^{-1 / 48}\left|{\widehat{v_{i}}}_{i}\left(q_{i}, k_{i}\right)\right| \\
& \quad\left\|\mathcal{F}^{-1}\left(\langle\sigma\rangle^{1 / 16}|\widehat{w}|\right)\right\|_{L^{4}\left(\mathbb{T}^{2}\right)}\left\|\mathcal{F}^{-1}\left(\langle\tilde{\sigma}\rangle^{1 / 16}\langle k\rangle^{-1 / 48}\left|\widehat{\overline{v_{1}}}\right|\right)\right\|_{L^{4}\left(\mathbb{T}^{2}\right)} \\
& \prod_{i=2}^{3}\left\|\mathcal{F}^{-1}\left(\langle\sigma\rangle^{1 / 16}\langle k\rangle^{-1 / 48}\left|\widehat{v}_{i}\right|\right)\right\|_{L^{4}\left(\mathbb{T}^{2}\right)} \\
& \lesssim\|w\|_{X^{7 / 16,0}} \prod_{i=1}^{3}\left\|v_{i}\right\|_{X^{7 / 16,-1 / 48}}
\end{aligned}
$$

where we used (8) and (14) in the last step.

Let $\left\{u_{0, n}\right\} \subset L^{2}(\mathbb{T})$ be a sequence converging weakly but not strongly to $u_{0}$ in $L^{2}(\mathbb{T})$ and let $\left\{u_{n}\right\}$ be the sequence of emanating solutions. By the BanachSteinhaus'theorem, $\left\{\left\|u_{0, n}\right\|_{L^{2}}\right\}$ is bounded in $\mathbb{R}_{+}$and thus admits at least one adherence value. Since we assume that $u_{0, n} \nrightarrow u_{0}$ in $L^{2}(\mathbb{T})$, one adherence value at least must be different from $\left\|u_{0}\right\|_{L^{2}}$. Let us denote by $\alpha \geq 0$ such an adherence value of $\left\{\left\|u_{0, n}\right\|_{L^{2}}\right\}$ and let $\left\{\left\|u_{0, n_{k}}\right\|_{L^{2}}\right\}$ be any subsequence converging towards $\alpha$. From Theorem 2.1 we know that the corresponding subsequence of solutions $\left\{u_{n_{k}}\right\}$ is bounded in $X_{1}^{1 / 2,0}$ and thus, up to the extraction of a subsequence, converges weakly to some 
$v$ in $X_{1}^{1 / 2,0}$. Moreover, since the $L^{2}$-norm is conserved for $u_{n}$, we infer that

$$
\begin{aligned}
u_{n}(t)= & V(t) u_{0, n}-\gamma \int_{0}^{t} V\left(t-t^{\prime}\right)\left(\Lambda_{1}\left(u_{n}\left(t^{\prime}\right)\right)+\Lambda_{2}\left(u_{n}\left(t^{\prime}\right)\right) d t^{\prime}\right. \\
& \left.-\frac{\gamma}{\pi}\left\|u_{0, n}\right\|_{L^{2}}^{2} \int_{0}^{t} V\left(t-t^{\prime}\right) u_{n}\left(t^{\prime}\right) d t^{\prime}, \forall t \in\right]-1,1[.
\end{aligned}
$$

From the linear estimates (6)-(8), Lemmas 2.2 and 2.3 and the above convergence results, it follows that

$$
\begin{aligned}
v(t)= & V(t) u_{0}-\gamma \int_{0}^{t} V\left(t-t^{\prime}\right)\left(\Lambda_{1}\left(v\left(t^{\prime}\right)\right)+\Lambda_{2}\left(v\left(t^{\prime}\right)\right) d t^{\prime}\right. \\
& -\frac{\gamma}{\pi} \alpha^{2} \int_{0}^{t} V\left(t-t^{\prime}\right) v\left(t^{\prime}\right) d t^{\prime}
\end{aligned}
$$

and $v$ is solution of the following Cauchy problem on $]-1,1[$ :

$$
\left\{\begin{array}{l}
i v_{t}+v_{x x}+\gamma\left(\Lambda_{1}+\Lambda_{2}\right)(v)+\frac{\gamma}{\pi} \alpha^{2} v=0 \\
v(0)=u_{0}
\end{array} .\right.
$$

Proceeding exactly as for the cubic Schrödinger equation, it is easy to prove that this Cauchy problem is globally well-posed ${ }^{2}$ in $H^{s}(\mathbb{T}), s \geq 0$, with a solution belonging for all $T>0$ to

$$
C\left([-T, T] ; H^{s}(\mathbb{T})\right) \cap L^{4}(]-T, T[\times \mathbb{T}) \cap X_{T}^{1 / 2,0}
$$

with uniqueness in $L^{4}(]-T, T[\times \mathbb{T})$. Therefore, there exists only one possible limit and thus the whole sequence $\left\{u_{n_{k}}\right\}$ converges weakly to $v$ in $X_{1}^{1 / 2,0}$. Moreover, using the equation satisfied by the $u_{n}$ and the uniform bound in $L^{\infty}(]-T, T\left[; L^{2}(\mathbb{T})\right) \cap$ $L^{4}(]-T, T[\times \mathbb{T})$, it is easy to check that for any time-independent $2 \pi$-periodic smooth function $\phi$, the family $\left\{t \mapsto\left(u_{n_{k}}(t), \phi\right)_{L^{2}}\right\}$ is bounded in $C([-1,1])$ and uniformly equi-continuous on $[-1,1]$. Ascoli's theorem then ensures that $\left(u_{n_{k}}, \phi\right)$ converges to $(v, \phi)$ on $[-1,1]$ and thus $u_{n_{k}}(t) \rightarrow v(t)$ in $L^{2}(\mathbb{T})$ for all $t \in[-1,1]$. By direct iteration this clearly also holds for all $t \in \mathbb{R}$.

Note that, since the $L^{2}$-norm of the solution is preserved by the flow of (19), $v$ can be also characterized as the unique solution in $L^{4}(]-T, T[\times \mathbb{T})$ to

$$
\left\{\begin{array}{l}
i v_{t}+v_{x x}+\gamma|v|^{2} v+\frac{\gamma}{\pi}\left(\alpha^{2}-\left\|u_{0}\right\|_{L^{2}}^{2}\right) v=0 \\
v(0)=u_{0}
\end{array} .\right.
$$

Finally, setting

$$
\tilde{v}(t, x)=\exp \left(-\frac{i \gamma}{\pi}\left(\alpha^{2}-\left\|u_{0}\right\|_{L^{2}}^{2}\right) t\right) v(t, x)
$$

we notice that $\tilde{v} \in L^{4}(]-T, T[\times \mathbb{T})$ and satisfies (1) with $\tilde{v}(0)=u_{0}$. By uniqueness (see Theorem 2.1), it follows that $\tilde{v}=u$, where $u$ is the solution to (1) emanating from $u_{0}$. This completes the proof of the theorem.

\footnotetext{
${ }^{2}$ Note that the $L^{2}$-norm is preserved by the flow of (19)
} 


\section{Acknowledgements}

The author was partially supported by the ANR project " Etude qualitative des EDP dispersives".

\section{References}

[1] M. Ablowitz and Y. Ma, The periodic nonlinear Schrödinger equation, Stud. Appl. Math. 65 (1981) 113-158.

[2] I. Bejenaru and T. Tao, Sharp well-posedness and ill-posedness results for a quadratic non-linear Schrödinger equation, J. Funct. Anal. 233 (2006) 228-259.

[3] J. Bourgain, Fourier transform restriction phenomena for certain lattice subsets and application to nonlinear evolution equations I. The Schrödinger equation, GAFA 3 (1993) 157-178.

[4] _ Periodic Korteweg de Vries equation with measures as initial data, Sel. Math. New. Ser. 3 (1993) 115-159.

[5] N. Burq, P. Gérard, and N. Tzvetkov, An instability property of the nonlinear Schrödinger equation on $S^{d}$, Math. Res. Lett. 9 (2002), no. 2-3, 323-335.

[6] M. Christ, J. Colliander, and T. Tao, Asymptotics, frequency modulation, and low regularity ill-posedness for canonical defocusing equations, Amer. J. Math. 125 (2003), no. 6, 1235-1293.

[7] — Instability of the periodic nonlinear Schrödinger equation, preprint arXiv:math/0311048 (2003)

[8] O. Goubet and L. Molinet, Global attractor for weakly damped nonlinear Schrödinger equations in $L^{2}(\mathbb{R})$, preprint $(2007)$

[9] C. E. Kenig, G. Ponce, and L. Vega, On the ill-posedness of some canonical dispersive equations, Duke Math. J. 106 (2001), no. 3, 617-633.

[10] Y. Tsutsumi, $L^{2}$-solutions for nonlinear Schrödinger equations and nonlinear groups, Funk. Ekva. 30 (1987) 115-125.

LAGA, Institut Galilée, Université Paris 13, 93430 Villetaneuse, FRAnCE 